Canadian

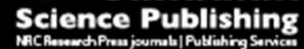

Canadian Journal of Chemistry Revue canadienne de chimie

\title{
Pyridine, Thiophosphine and Selenophosphine Complexes of the Phenylphosphine Dication
}

\begin{tabular}{|r|l|}
\hline Journal: & Canadian Journal of Chemistry \\
\hline Manuscript ID & cjc-2017-0596.R1 \\
\hline Danuscript Type: & Article \\
\hline Complete List of Authors: & $\begin{array}{l}\text { Sinclair, Hannah; University of Victoria, Department of Chemistry } \\
\text { Suter, Riccardo; University of Victoria, Department of Chemistry } \\
\text { Burford, Neil; University of Victoria, Department of Chemistry } \\
\text { McDonald, Robert; University of Alberta, Chemistry } \\
\text { Ferguson, Michael; University of Alberta }\end{array}$ \\
\hline $\begin{array}{r}\text { Is the invited manuscript for } \\
\text { consideration in a Special } \\
\text { Issue?: }\end{array}$ & Dalhousie \\
\hline Keyword: & $\begin{array}{l}\text { phosphorus, cations, coordination complexes, synthesis, molecular } \\
\text { structure }\end{array}$ \\
\hline & \multicolumn{2}{|c}{} \\
\hline
\end{tabular}




\section{Pyridine, Thiophosphine and Selenophosphine Complexes of the Phenylphosphine Dication}

Hannah Sinclair, ${ }^{\mathrm{a}}$ Riccardo Suter, ${ }^{\mathrm{a}}$ Neil Burford, ${ }^{\mathrm{a}}$ Robert McDonald, ${ }^{\mathrm{b}}$ and Michael J. Ferguson ${ }^{\mathrm{b}}$

${ }^{a}$ Department of Chemistry, University of Victoria, Victoria, British Columbia, V8W 3V6, Canada;

nburford@uvic.ca; 250-721-7150; 250-721-7147

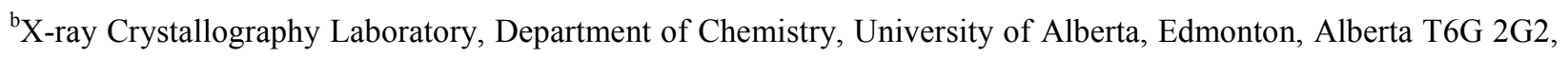
Canada;

CCDC 1560524-1560527 and 1570485 contains the supplementary crystallographic data for this paper. The data can be obtained free of charge from The Cambridge Crystallographic Data Centre via www.ccdc.cam.ac.uk/structures. 


\begin{abstract}
Compounds of the generic formula $[\mathrm{PhPL}][\mathrm{OTf}]_{2}$ with $\mathrm{L}=$ bipyridine (bipy) and 4,4'-di(tert-butyl)-2,2'-bipyridine (Bbipy), and $\left[\mathrm{PhPL}_{2}\right][\mathrm{OTf}]_{2}$ with $\mathrm{L}=$ 4-dimethylaminopyridine (dmap), tricyclohexyl-thiophosphine and tricyclohexyl-selenophosphine have been prepared by the reaction of dichlorophenylphosphine with two equivalents of trimethylsilyl triflate and the respective ligand. The new complexes of the phenylphosphine dication with this variety of ligands expands the scope of coordination complexes involving phosphorus as an acceptor.
\end{abstract}

Key words: phosphorus, cations, coordination complexes, synthesis, molecular structure 


\section{Introduction}

Much of the chemistry of phosphorus in a formal oxidation state of III involves the phosphorus centre behaving as a Lewis base or ligand. Imposition of a cationic charge on a molecule containing a phosphine centre enables Lewis acid behavior that diversifies the chemistry of phosphorus. Complexes of $\mathrm{RNP}^{+}(\mathbf{1})^{[1]}, \mathrm{R}_{2} \mathrm{P}^{+}(\mathbf{2})^{[2]}, \mathrm{RP}^{2+}(\mathbf{3})^{[2 \mathrm{a}}$ ${ }^{2 b, 3]}, \mathrm{R}_{3} \mathrm{P}_{-} \mathrm{PR}_{3}{ }^{2+}(\mathbf{4})^{[4]}$ and $\mathrm{P}^{3+}(\mathbf{5})^{[5]}$ have been reported with a variety of ligands. ${ }^{[6]}$ In addition, bipyridine complexes of $\mathrm{P}^{3+}(\mathbf{6})^{[7]}$ illustrate the potentially versatile coordination chemistry of phosphorus. We have now expanded the ligand scope for derivatives of $\mathbf{3}$ for $\mathrm{R}=\mathrm{Ph}$. Complexes of $[\mathrm{PhP}]^{2+}$ as trifluoromethanesulfonate (triflate) salts with 2,2'-bipyridine $\quad\left\{[\mathrm{PhP}(\right.$ bipy $\left.)][\mathrm{OTf}]_{2}\right\}, \quad 4,4$ '-di(tert-butyl)-2,2'-bipyridine $\quad\left\{[\mathrm{PhP}(\mathrm{Bbipy})][\mathrm{OTf}]_{2}\right\}, \quad 4-$ dimethylaminopyridine $\quad\left\{\left[\mathrm{PhP}(\mathrm{dmap})_{2}\right][\mathrm{OTf}]_{2}\right\}$, thiophosphine $\quad\left\{\left[\mathrm{PhP}\left(\mathrm{SPCy}_{3}\right)_{2}\right][\mathrm{OTf}]_{2}\right\}$ and selenophosphine $\left\{\left[\mathrm{PhP}\left(\mathrm{SePCy}_{3}\right)_{2}\right][\mathrm{OTf}]_{2}\right\}$ have been prepared and comprehensively characterized. The compounds represent new derivatives of chelate complexes of phosphorus and the first examples of pyridine, thio- and seleno- complexes of phosphine dications.

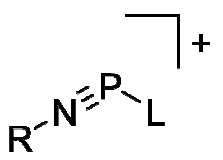

1<smiles>[R]P([3H])CC</smiles>

3<smiles>[3H]I([3H])[18O]CC</smiles>

5

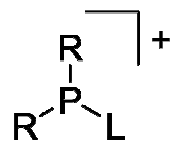

2

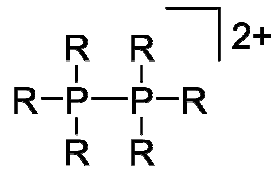

4

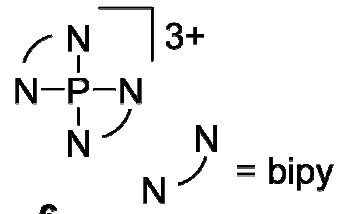

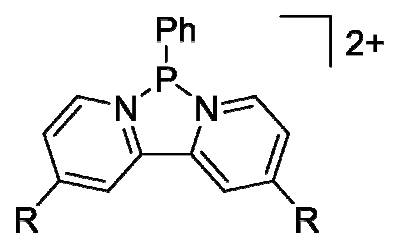

$[\mathrm{PhP}(\text { bipy })]^{2+} \quad(\mathrm{R}=\mathrm{H})$

$[\mathrm{PhP}(\text { Bbipy })]^{2+} \quad\left(\mathrm{R}={ }^{\mathrm{t}} \mathrm{Bu}\right)$

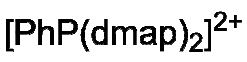

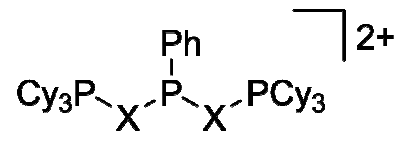

$\left[\mathrm{PhP}\left(\mathrm{SPCy}_{3}\right)_{2}\right]^{2+} \quad(\mathrm{X}=\mathrm{S})$ $\left[\mathrm{PhP}\left(\mathrm{SePCy}_{3}\right)_{2}\right]^{2+} \quad(\mathrm{X}=\mathrm{Se})$ 


\section{Results and Discussion}

$\mathrm{PhPCl}_{2}$ reacts with TMSOTf in the presence of bipy or Bbipy, yielding one new signal in the ${ }^{31} \mathrm{P}\left\{{ }^{1} \mathrm{H}\right\}$ NMR spectra of the reaction mixtures $(\delta=136.8 \mathrm{ppm}$ for bipy and $133.8 \mathrm{ppm}$ for Bbipy). Clean products and optimal reaction conditions are obtained with a small excess of TMSOTf ( 2.5 equivalents), which can be removed by precipitating the product from either DCM or acetonitrile, and washing the solid with diethyl ether. Analogous complexes have been formed following a similar synthetic procedures; $\left[\mathrm{PhP}(\mathrm{dmap})_{2}\right][\mathrm{OTf}]_{2} \quad(\delta=121.0 \mathrm{ppm}),\left[\mathrm{PhP}\left(\mathrm{SPCy}_{3}\right)_{2}\right][\mathrm{OTf}]_{2}$ $(\delta=61.5 \mathrm{ppm})$ or $\left[\mathrm{PhP}\left(\mathrm{SePCy}_{3}\right)_{2}\right][\mathrm{OTf}]_{2}(\delta=48.9 \mathrm{ppm}) .{ }^{[8]}$ After stirring the reaction mixtures at room temperature for at least one hour, the volatiles were removed under reduced pressure to provide colourless solids. Derivatives of $\left[\mathrm{PhPL}_{\mathrm{x}}\right][\mathrm{OTf}]_{2}$ have been crystallized from acetonitrile or DCM solutions layered with diethyl ether and the solid state structures have been determined by X-ray crystallography. The ${ }^{31} \mathrm{P}\left\{{ }^{1} \mathrm{H}\right\}$ NMR spectrum for derivatives of $\left[\mathrm{PhP}\left(\mathrm{XPCy}_{3}\right)_{2}\right]^{2+}$ exhibit a doublet and triplet resonance consistent with a $\mathrm{R}_{3} \mathrm{P}-\mathrm{X}-\mathrm{PR}-\mathrm{X}-\mathrm{PR}_{3}$ chain (Table 1). The ${ }^{2} J_{\mathrm{P}, \mathrm{P}}$ values are typical, and the doublet of doublets resonance in the ${ }^{77} \mathrm{Se}\left\{{ }^{1} \mathrm{H}\right\}$ NMR spectrum $\left(\delta=119.2, d d, J_{\text {SeP }}=\right.$ 416.5, $133.5 \mathrm{~Hz})$ for $\left[\mathrm{PhP}\left(\mathrm{SePCy}_{3}\right)_{2}\right]^{2+}$ are consistent with the observed solid state structure. The $\mathrm{PhP}$ centres of $\left[\mathrm{PhP}\left(\mathrm{XPCy}_{3}\right)_{2}\right]^{2+}$ resonate at significantly lower frequency than those involving pyridine ligands. The tricyclohexylphosphine units on the other hand resonate at higher frequency compared to $\left[\mathrm{HPCy}_{3}\right][\mathrm{OTf}](\delta=29.5$ $\mathrm{ppm})^{[9]}$ or the free ligand $\left(\mathrm{SPCy}_{3} \delta=62.4 \mathrm{ppm}\right) \cdot{ }^{[10]}$ The ${ }^{19} \mathrm{~F}$ chemical NMR shift for the triflate anions is between 78.8 and $79.2 \mathrm{ppm}$ and indicates free triflate in solution with weak to no interaction with the phosphorus dication.

Table 1: ${ }^{31} \mathrm{P}\left\{{ }^{1} \mathrm{H}\right\}-\mathrm{NMR}$ data for the dicationic species $\left[\mathrm{PPhL} \mathrm{L}_{\mathrm{x}}\right]^{2+}$

\begin{tabular}{lll}
\hline & ${ }^{31} \mathrm{P}\left\{{ }^{1} \mathrm{H}\right\}[\delta \mathrm{ppm}]$ & ${ }^{2} J_{\mathrm{PP}}[\mathrm{Hz}]$ \\
\hline$[\mathrm{PhP}(\text { bipy })]^{2+}$ & $136.8(\mathrm{~s})$ & - \\
{$[\mathrm{PhP}(\text { Bbipy })]^{2+}$} & $133.8(\mathrm{~s})$ & - \\
{$\left[\mathrm{PhP}(\text { dmap })_{2}\right]^{2+}$} & $121.0(\mathrm{~s})$ & - \\
{$\left[\mathrm{PhP}\left(\mathrm{SPCy}_{3}\right)_{2}\right]^{2+}$} & $73.7(\mathrm{~d}), 61.5(\mathrm{t})$ & 21 \\
{$\left[\mathrm{PhP}\left(\mathrm{SePCy}_{3}\right)_{2}\right]^{2+}$} & $72.3(\mathrm{~d}), 48.9(\mathrm{t})$ & 26 \\
\hline
\end{tabular}


Table 2: Crystallographic data for $[\mathrm{PhP}($ bipy $)][\mathrm{OTf}]_{2,}[\mathrm{PhP}(\mathrm{Bbipy})][\mathrm{OTf}]_{2,}\left[\mathrm{PhP}(\mathrm{dmap})_{2}\right][\mathrm{OTf}]_{2}$,

$\left[\mathrm{PhP}\left(\mathrm{SPCy}_{3}\right)_{2}\right][\mathrm{OTf}]_{2,}\left[\mathrm{PhP}\left(\mathrm{SePCy}_{3}\right)_{2}\right][\mathrm{OTf}]_{2}$

\begin{tabular}{|c|c|c|c|c|c|}
\hline & $\begin{array}{c}{[\mathrm{PhP}(\text { bipy })]} \\
{[\mathrm{OTf}]_{2}}\end{array}$ & $\begin{array}{c}{[\mathrm{PhP}(\text { Bbipy })]} \\
{[\mathrm{OTf}]_{2}}\end{array}$ & $\begin{array}{c}\left.\mathrm{PhP}(\mathrm{dmap})_{2}\right] \\
{[\mathrm{OTf}]_{2}}\end{array}$ & $\begin{array}{c}{\left[\mathrm{PhP}\left(\mathrm{SPCy}_{3}\right)_{2}\right]} \\
{[\mathrm{OTf}]_{2}}\end{array}$ & $\begin{array}{c}{\left[\mathrm{PhP}\left(\mathrm{SePCy}_{3}\right)_{2}\right]} \\
{[\mathrm{OTf}]_{2}}\end{array}$ \\
\hline $\begin{array}{l}\text { Empirical } \\
\text { formula }\end{array}$ & $\mathrm{C}_{18} \mathrm{H}_{13} \mathrm{~N}_{2} \mathrm{O}_{6} \mathrm{~F}_{6} \mathrm{PS}_{2}$ & $\mathrm{C}_{26} \mathrm{H}_{29} \mathrm{~N}_{2} \mathrm{O}_{6} \mathrm{~F}_{6} \mathrm{PS}_{2}$ & $\mathrm{C}_{29} \mathrm{H}_{35} \mathrm{~N}_{6} \mathrm{O}_{6} \mathrm{~F}_{6} \mathrm{PS}_{2}$ & $\mathrm{C}_{44} \mathrm{H}_{71} \mathrm{~F}_{6} \mathrm{O}_{6} \mathrm{P}_{3} \mathrm{~S}_{4}$ & $\mathrm{C}_{44} \mathrm{H}_{71} \mathrm{O}_{6} \mathrm{~F}_{6} \mathrm{P}_{3} \mathrm{~S}_{2} \mathrm{Se}_{2}$ \\
\hline Formula weight & 562.39 & 674.60 & 772.72 & 1031.15 & 1124.95 \\
\hline Temperature/K & 173.15 & 173.15 & 173.15 & 173.15 & 193.15 \\
\hline Crystal system & triclinic & triclinic & orthorhombic & orthorhombic & orthorhombic \\
\hline Space group & $\mathrm{P}-1$ & $\mathrm{P}-1$ & Fdd2 & $\mathrm{Pca}_{1}$ & $\mathrm{Pca}_{1}$ \\
\hline $\mathrm{a} / \AA$ & $7.3772(2)$ & $10.3407(7)$ & $20.5652(4)$ & $19.3394(6)$ & $19.1271(10)$ \\
\hline $\mathrm{b} / \AA$ & $11.0469(3)$ & $10.9356(7)$ & $34.7966(7)$ & $10.6524(4)$ & $21.3744(11)$ \\
\hline$c / \AA$ & $14.8136(5)$ & $14.1649(9)$ & $16.2609(3)$ & $24.1489(8)$ & $24.5753(12)$ \\
\hline$\alpha^{\circ}$ & $74.9952(18)$ & $109.229(3)$ & 90 & 90 & 90 \\
\hline$\beta /{ }^{\circ}$ & $77.2568(15)$ & $97.623(3)$ & 90 & 90 & 90 \\
\hline$\gamma /{ }^{\circ}$ & $85.2235(14)$ & $93.345(2)$ & 90 & 90 & 90 \\
\hline Volume $/ \AA^{3}$ & $1136.91(6)$ & $1490.12(17)$ & $11636.3(4)$ & 4974.9(3) & 10047.1(9) \\
\hline Z & 2 & 2 & 16 & 4 & 8 \\
\hline$\rho_{\text {calc }} \mathrm{g} / \mathrm{cm}^{3}$ & 1.643 & 1.504 & 1.764 & 1.377 & 1.487 \\
\hline$\mu / \mathrm{mm}^{-1}$ & 3.625 & 2.863 & 3.066 & 3.243 & 1.717 \\
\hline $\mathrm{F}(000)$ & 568.0 & 696.0 & 6400.0 & 2184.0 & 4656.0 \\
\hline $\begin{array}{l}\text { Reflections } \\
\text { collected }\end{array}$ & 7962 & 10609 & 19943 & 174874 & 82438 \\
\hline $\begin{array}{l}\text { Independent } \\
\text { reflections }\end{array}$ & 4427 & 5791 & 5724 & 9890 & 23072 \\
\hline $\mathrm{R}_{\mathrm{int}}$ & 0.0409 & 0.0260 & 0.0167 & 0.0431 & 0.0554 \\
\hline $\mathrm{R}_{\text {sigma }}$ & 0.0490 & 0.0334 & 0.0165 & 0.0185 & 0.0578 \\
\hline $\begin{array}{l}\text { Data/restraints/p } \\
\text { arameters }\end{array}$ & $4427 / 0 / 316$ & $5791 / 0 / 388$ & $5724 / 1 / 374$ & $9890 / 1 / 568$ & $23072 / 1 / 1136$ \\
\hline GOF & 1.084 & 1.052 & 1.078 & 0.998 & 1.006 \\
\hline
\end{tabular}




$\begin{array}{llllll}\mathrm{R}_{1}[\mathrm{I}>2 \sigma(\mathrm{I})] & 0.0503, & 0.0378, & 0.0513, & 0.0230, & 0.0399, \\ \mathrm{wR}_{2}[\mathrm{I}>2 \sigma(\mathrm{I})] & 0.1404 & 0.1057 & 0.1527 & 0.0616 & 0.0822 \\ \text { Largest diff. } & & & & \\ \text { peak/hole } / \mathrm{e} \AA^{-3} & 0.75 /-0.56 & 0.49 /-0.41 & 1.64 /-0.46 & 0.22 /-0.32 & 1.30 /-0.93\end{array}$

As shown in Figure 1, each of the solid state structures contains a $\mathrm{PhP}$ unit in which the phosphorus centre is attached to two nitrogen, sulfur or selenium atoms. The pyridine derivatives show long interactions with oxygen atoms from the triflate anions (Table 3). The structure of $\left[\mathrm{PhP}(\right.$ bipy) $][\mathrm{OTf}]_{2}$ involves a dimeric arrangement with two triflate anions linking phosphorus centres. All P---O interactions are outside the sum of the P-O covalent radii $\left(\Sigma_{\mathrm{r}, \text { cov }}=1.74 \AA\right),{ }^{[1]}$ but some are inside the sum of the van der Waals radii $\left(\Sigma_{\mathrm{r}, \mathrm{ddW}}=3.32 \AA\right) .{ }^{[12]}$ The N-P bond lengths in $\left[\mathrm{PPh}(\mathrm{dmap})_{2}\right]^{2+}$ are indistinguishable and are significantly shorter than the N-P bond lengths in $[\mathrm{PhP}($ bipy $)][\mathrm{OTf}]_{2}$ and $[\mathrm{PhP}(\mathrm{Bbipy})][\mathrm{OTf}]_{2}$, consistent with the greater basicity of dmap. 
b)

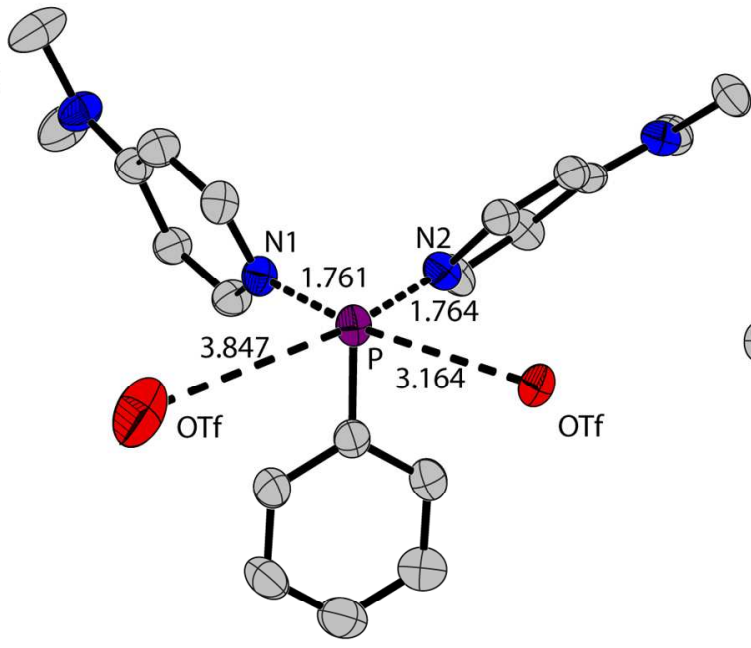

a)

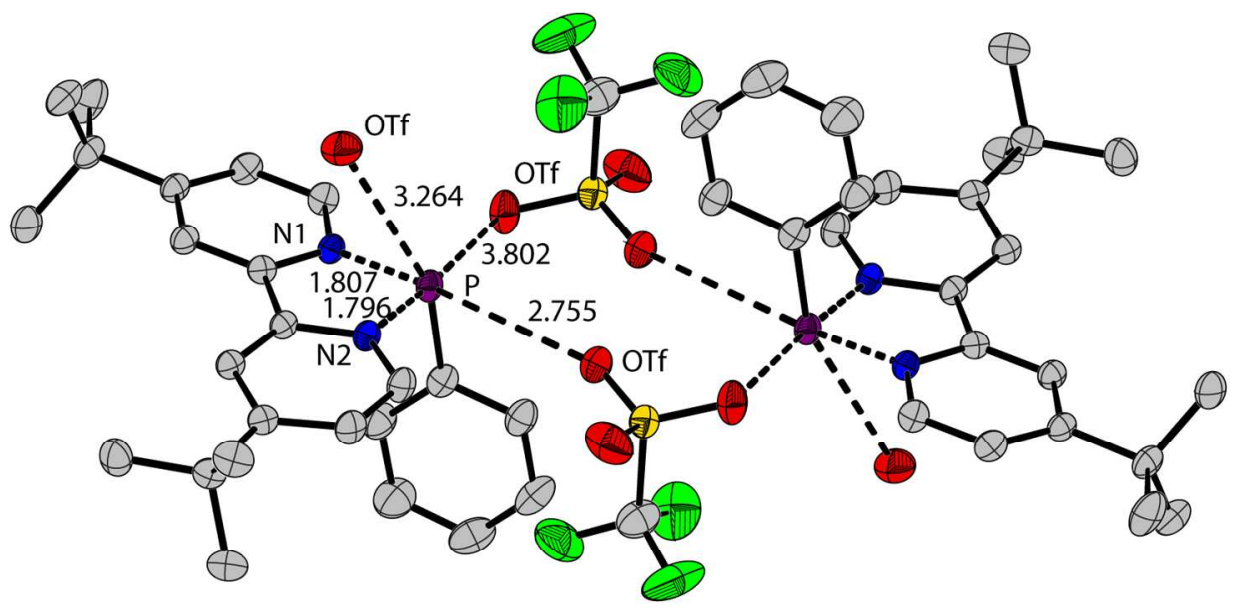

c)

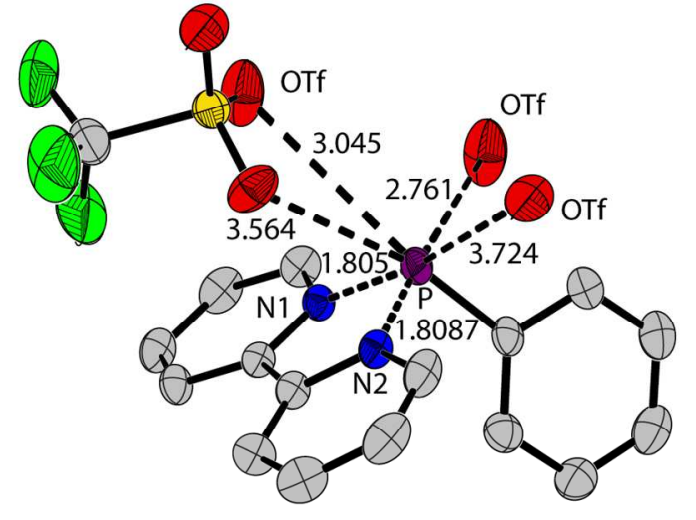

d)
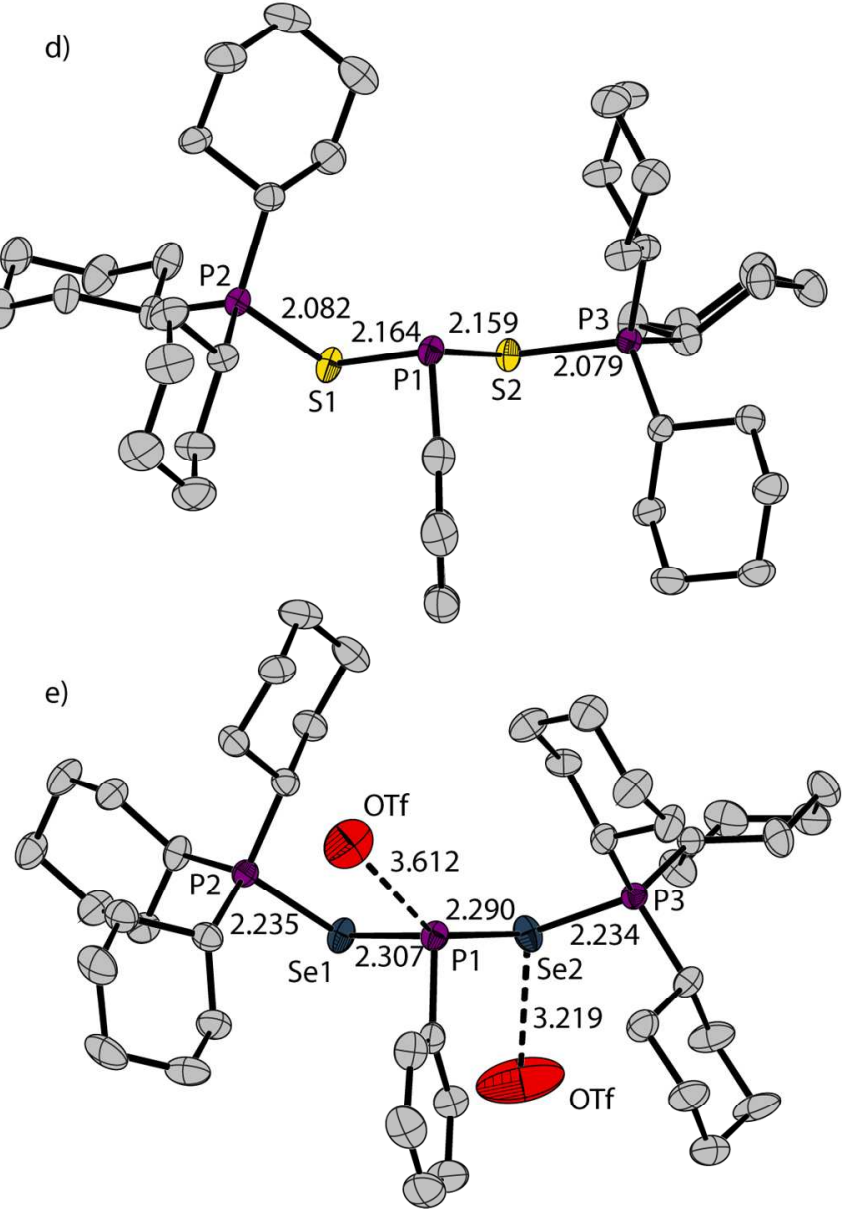

Figure 1: Solid state structures of the cations in (a) $\left[\{\mathrm{PhP}(\text { Bbipy })\}_{2}\right][\mathrm{OTf}]_{4}$, (b) $\left[\mathrm{PhP}(\mathrm{dmap})_{2}\right][\mathrm{OTf}]_{2}$, (c) $[\mathrm{PhP}($ bipy $)][\mathrm{OTf}]_{2}$, (d) $\left[\mathrm{PhP}\left(\mathrm{SPCy}_{3}\right)_{2}\right][\mathrm{OTf}]_{2}$, (e) $\left[\mathrm{PhP}\left(\mathrm{SePCy}_{3}\right)_{2}\right][\mathrm{OTf}]_{2}$. Hydrogen atoms are omitted for clarity, if not required for understanding only the coordinating oxygen atoms for the triflate anions are shown. Thermal ellipsoids are shown at the $50 \%$ probability level, interatomic distances in $\AA$. 
Complexes of $\mathrm{PhP}^{2+}$ with phosphine chalcogenide ligands represent $\mathrm{R}_{3} \mathrm{P}-\mathrm{X}-\mathrm{PR}-\mathrm{X}-\mathrm{PR}_{3}$ chains $(\mathrm{X}=\mathrm{S}$ or $\mathrm{Se})$. In the solid state structure of $\left[\mathrm{PhP}\left(\mathrm{SPCy}_{3}\right)_{2}\right][\mathrm{OTf}]_{2}$ the closest interactions with the triflate anion are outside the sum of the

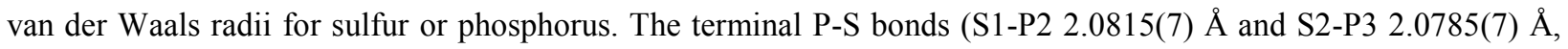
respectively) are significantly longer than in $\mathrm{SPCy}_{3}(1.966(5) \AA),{ }^{[13]}$ but are shorter than the central bonds (S1-P1 2.1638(7) $\AA$ and S2-P1 2.1586(7) $\AA$ ). Interestingly, the S-P bond distances in the neutral carbon analogue $\mathrm{Ph}_{3} \mathrm{C}-\mathrm{S}$ $\mathrm{PPh}-\mathrm{S}_{-} \mathrm{CPh}_{3}$ (2.122(1) and 2.120(1) $\AA$, respectively) are only slightly shorter. ${ }^{[14]}$ The solid state structure of $\left[\mathrm{PhP}\left(\mathrm{SePCy}_{3}\right)_{2}\right][\mathrm{OTf}]_{2}$ reveals P-Se bond lengths (P2-Se1 2.2350(13) and P3-Se2 2.2342(12) $\AA$ ) that are consistent with the trends in the sulfur analogue in comparison with $\mathrm{SePCy}_{3}(\mathrm{P}-\mathrm{Se} 2.108(1) \AA),{ }^{[15]}$ although the differences are smaller. In comparison, $\left[\mathrm{Ph}_{3} \mathrm{P}-\mathrm{SePh}\right] \mathrm{Br}$ exhibits a Se-P bond distance of $2.2260(16) \AA{ }^{[16]}$ which is significantly shorter than those in $\left[\mathrm{PhP}\left(\mathrm{SePCy}_{3}\right)_{2}\right][\mathrm{OTf}]_{2}$.

Table 3: Selected structural parameters for $\left[\mathrm{PPh}(\mathrm{dmap})_{2}\right][\mathrm{OTf}]_{2},[\mathrm{PPh}(\mathrm{Bbipy})][\mathrm{OTf}]_{2}$, and $[\mathrm{PPh}($ bipy $)][\mathrm{OTf}]_{2}$ compared to the chalcogen derivatives $\left[\mathrm{PhP}\left(\mathrm{SPCy}_{3}\right)_{2}\right][\mathrm{OTf}]_{2}$ and $\left[\mathrm{PhP}\left(\mathrm{SePCy}_{3}\right)_{2}\right][\mathrm{OTf}]_{2}$. Bond distances in $\AA$ and sum of angles around the phosphorus dication $\left(\Sigma_{\mathrm{P}}\right)$ in ${ }^{\circ} \mathrm{X}=\mathrm{S}$ or Se, respectively.

\begin{tabular}{ccccccc}
\hline ligand & dmap & Bbipy & bipy & & $\mathrm{SPCy}_{3}$ & $\mathrm{SePCy}_{3}$ \\
\hline P1-N1 & $1.761(4)$ & $1.7962(13)$ & $1.8087(19)$ & $\mathrm{P} 1-\mathrm{X} 1$ & $2.1638(7)$ & $2.3066(13)$ \\
P1-N2 & $1.764(4)$ & $1.8069(14)$ & $1.805(2)$ & $\mathrm{P} 1-\mathrm{X} 2$ & $2.1586(7)$ & $2.2903(13)$ \\
P1-C1 & $1.813(5)$ & $1.8182(17)$ & $1.805(2)$ & $\mathrm{P} 1-\mathrm{C} 1$ & $1.814(2)$ & $1.181(5)$ \\
& & & & P2-X1 & $2.0815(7)$ & $2.2350(13)$ \\
& & & & P3-X2 & $2.0785(7)$ & $2.2342(12)$ \\
P-OTf & 3.847 & 2.755 & 2.761 & P-OTf & - & 3.612 \\
& 3.164 & 3.264 & 3.045 & X-OTf & - & 3.219 \\
& & 3.802 & 3.564 & & & \\
$\Sigma_{\mathrm{P}}^{3}$ & 300.7 & 280.3 & 283.7 & & & \\
\end{tabular}

\section{Conclusion:}

We have expanded the scope of ligands that engage the phenylphosphine dication, to include dmap, bipy, Bbipy, $\mathrm{SPCy}_{3}$ and $\mathrm{SePCy}_{3}$ and showed the potential of $\mathrm{PhP}^{2+}$ being a Lewis acceptor. Such cationic complexes may be further applied as ligands for transition metals or as active species in Lewis acid catalysed organic transformations. 


\section{Acknowledgements}

We thank the Natural Sciences and Engineering Research Council of Canada for funding.

\section{Experimental}

All reagents were handled in an Innovative Technology, inc. System One Glovebox under an atmosphere of dry $\mathrm{N}_{2}$. Solvents were dried over $\mathrm{Na}\left(\mathrm{Et}_{2} \mathrm{O}\right)$ or $\mathrm{CaH}_{2}\left(\mathrm{CH}_{2} \mathrm{Cl}_{2}\right)$ and then distilled. Anhydrous grade $\mathrm{MeCN}$ was obtained from Sigma-Aldrich and used without purification. Prior to use, all solvents were stored for at least $48 \mathrm{hr}$ over $3 \AA$ molecular sieves $(\mathrm{MeCN})$ or $4 \AA$ molecular sieves $\left(\mathrm{Et}_{2} \mathrm{O}\right.$ and $\left.\mathrm{CH}_{2} \mathrm{Cl}_{2}\right)$, which had been activated at $300{ }^{\circ} \mathrm{C}$ under dynamic vacuum for $48 \mathrm{hr}$. Deuterated solvents were dried over their corresponding size molecular sieves and were stored for at least $48 \mathrm{hr}$. TMSOTf (99\%) was distilled before use. 4-Dimethylaminopyridine (dmap) was purified by sublimation under vacuum. $\mathrm{SPCy}_{3}$ and $\mathrm{SePCy}_{3}$ were synthesized according to literature procedures. ${ }^{17}$ Bipy and Bbipy were obtained from Sigma-Aldrich and used without further purification.

Reactions were carried out inside the glovebox in screw-cap glass vials that had been dried at $250{ }^{\circ} \mathrm{C}$ for at least $1 \mathrm{hr}$ and placed under dynamic vacuum (glovebox antechamber) while still hot. NMR spectra were obtained on a Bruker AVANCE $300 \mathrm{MHz}$ or $360 \mathrm{MHz}$ NMR Spectrometer, field strength is given explicitly with the characterization data for the compounds. ${ }^{1} \mathrm{H},{ }^{31} \mathrm{P}\left\{{ }^{1} \mathrm{H}\right\}$, and ${ }^{19} \mathrm{~F}\left\{{ }^{1} \mathrm{H}\right\}$ NMR chemical shifts were referenced to $\mathrm{SiMe}_{4}, 85 \% \mathrm{H}_{3} \mathrm{PO}_{4}$, and $\mathrm{CFCl}_{3}$, respectively. Infrared spectra were collected on samples prepared as nujol mulls between $\mathrm{NaCl}$ plates using a Perkin-Elmer Frontier FT-IR spectrometer. Peaks are reported in wavenumbers $\left(\mathrm{cm}^{-1}\right)$ with intensities (strong, medium, weak) in parentheses, relative to the most intense peak. Melting points were obtained on samples greasesealed in glass capillaries under dry nitrogen using an electrothermal apparatus. Unless otherwise stated, crystals for single crystal X-ray diffraction studies were obtained from slow diffusion of a layered non-solvent into a saturated solution of the compound. Single crystal X-ray diffraction data were collected on a Bruker D8/APEX II CCD diffractometer at $173 \mathrm{~K}$. All structures were solved by direct methods and refined by full-matrix least squares on $F^{2}$ (SHELXL-97 or SHELXL-2013). ${ }^{[17]}$ All non-hydrogen atoms were refined anisotropically while hydrogen atoms were assigned positions based on the $\mathrm{sp}^{2}$ or $\mathrm{sp}^{3}$ hybridization geometries of their attached carbons, and were given thermal parameters $20 \%$ greater than those of their parent atoms. 
Synthesis of $[\mathbf{P h P}(\mathbf{b i p y})][\mathrm{OTf}]_{2}$ : To a rapidly stirring solution of $\mathrm{PhPCl}_{2}(1.00 \mathrm{mmol}, 0.179 \mathrm{~g})$ and TMSOTf ( $2.00 \mathrm{mmol}, 0.445 \mathrm{~g})$ in $5 \mathrm{~mL}$ DCM, bipy $(1.00 \mathrm{mmol}, 0.268 \mathrm{~g})$ was added, producing a pale-yellow solution. The solution was stirred for $1 \mathrm{hr}$ and the solvent was removed under reduced pressure. The resulting white powder was washed with $\mathrm{Et}_{2} \mathrm{O}$ three times and was crystallized from $\mathrm{MeCN}$ layered with diethyl ether at $-26{ }^{\circ} \mathrm{C}$. Yield: $0.427 \mathrm{~g}$ (76 \%); mp: $218{ }^{\circ} \mathrm{C} \mathrm{dec} . ;{ }^{1} \mathrm{H}$ NMR $\left(\mathrm{CD}_{3} \mathrm{CN}, 298 \mathrm{~K}, 300 \mathrm{MHz}, \delta[\mathrm{ppm}]\right): 9.38-9.35\left(m, 2 \mathrm{H}, \mathrm{CH}_{\text {arom }}\right), 9.20(d t, 1.1$ $\left.\mathrm{Hz}, 8.3 \mathrm{~Hz}, 2 \mathrm{H}, \mathrm{CH}_{\text {arom }}\right), 9.00\left(t d, 1.2 \mathrm{~Hz}, 8.2 \mathrm{~Hz}, 2 \mathrm{H}, \mathrm{CH}_{\text {arom }}\right), 8.41-8.37\left(m, 2 \mathrm{H}, \mathrm{CH}_{\text {arom }}\right), 7.87-7.58(m, 5 \mathrm{H}$, $\left.\mathrm{CH}_{\text {arom }}\right) ;{ }^{13} \mathrm{C}\left\{{ }^{1} \mathrm{H}\right\}$ NMR $\left(\mathrm{CD}_{3} \mathrm{CN}, 298 \mathrm{~K}, 75.4 \mathrm{MHz}, \delta[\mathrm{ppm}]\right): 151.1(s), 146.3\left(d,{ }^{2} J_{C P}=16.2 \mathrm{~Hz}\right), 138.8(s), 136.3$ $\left(d, J_{C P}=31.3 \mathrm{~Hz}\right), 132.9\left(d,{ }^{3} J_{C P}=2.1 \mathrm{~Hz}\right), 131.8\left(d,{ }^{2} J_{C P}=11.6 \mathrm{~Hz}\right), 129.7(s), 129.3(s), 127.8\left(d,{ }^{2} J_{C P}=7 \mathrm{~Hz}\right)$; ${ }^{31} \mathrm{P}\left\{{ }^{1} \mathrm{H}\right\}$ NMR $\left(\mathrm{CD}_{3} \mathrm{CN}, 298 \mathrm{~K}, 121.4 \mathrm{MHz}, \delta[\mathrm{ppm}]\right): 136.8(s) ;{ }^{19} \mathrm{~F}\left\{{ }^{1} \mathrm{H}\right\} \mathrm{NMR}\left(\mathrm{CD}_{3} \mathrm{CN}, 298 \mathrm{~K}, 282.2 \mathrm{~Hz}, \delta\right.$ [ppm]): -79.2 (s); IR (Nujol mull, $\mathrm{NaCl}$ plates, $\left.\mathrm{cm}^{-1}\right): 1622(\mathrm{~m}), 1480(\mathrm{~m})$,

Synthesis of $[\mathbf{P h P}($ Bbipy $)][\mathrm{OTf}]_{2}$ : To a rapidly stirring solution of $\mathrm{PhPCl}_{2}(1.00 \mathrm{mmol}, 0.179 \mathrm{~g})$ and TMSOTf ( $2.00 \mathrm{mmol}, 0.445 \mathrm{~g})$ in $5 \mathrm{~mL}$ DCM, Bbipy $(1.00 \mathrm{mmol}, 0.268 \mathrm{~g})$ was added, producing a pale-yellow solution. The solution was stirred for $1 \mathrm{hr}$ and the solvent was removed under reduced pressure and washed with $\mathrm{Et}_{2} \mathrm{O}$ three times. The colourless powder was crystallized from $\mathrm{MeCN}$ layered with diethyl ether at $-26{ }^{\circ} \mathrm{C}$. Yield: $0.594 \mathrm{~g}(88 \%)$; mp: $230-231{ }^{\circ} \mathrm{C} ;{ }^{1} \mathrm{H}$ NMR $\left(\mathrm{CD}_{3} \mathrm{CN}, 298 \mathrm{~K}, 300 \mathrm{MHz}, \delta[\mathrm{ppm}]\right): 9.20-9.17\left(m, 4 \mathrm{H}, \mathrm{CH}_{\text {arom }}\right), 8.33(d t, 6.7 \mathrm{~Hz}, 1.9 \mathrm{~Hz}$, $\left.2 \mathrm{H}, \mathrm{CH}_{\text {arom }}\right), 7.85-7.58\left(m, 5 \mathrm{H}, \mathrm{CH}_{\text {arom }}\right), 1.55\left(s, 18 \mathrm{H}, \mathrm{CH}_{3}\right) ;{ }^{13} \mathrm{C}\left\{{ }^{1} \mathrm{H}\right\} \mathrm{NMR}\left(\mathrm{CD}_{3} \mathrm{CN}, 298 \mathrm{~K}, 75.4 \mathrm{MHz}, \delta[\mathrm{ppm}]\right)$ : $177.4(s), 146.2\left(d,{ }^{3} J_{C P}=4.2 \mathrm{~Hz}\right), 145.0\left(d,{ }^{2} J_{C P}=16.3 \mathrm{~Hz}\right), 138.4(s), 135.9\left(d, J_{C P}=31.4 \mathrm{~Hz}\right), 131.6\left(d,{ }^{2} J_{C P}=\right.$ $10.1 \mathrm{~Hz}), 130.4(s), 129.9\left(d,{ }^{3} J_{C P}=2.1 \mathrm{~Hz}\right), 125.4\left(d,{ }^{3} J_{C P}=4.3 \mathrm{~Hz}\right), 39.1(s), 30.4(s) ;{ }^{31} \mathrm{P}\left\{{ }^{1} \mathrm{H}\right\} \mathrm{NMR}\left(\mathrm{CD}_{3} \mathrm{CN}, 298\right.$ $\mathrm{K}, 121.4 \mathrm{MHz}, \delta[\mathrm{ppm}]): 133.8(s) ;{ }^{19} \mathrm{~F}\left\{{ }^{1} \mathrm{H}\right\} \mathrm{NMR}\left(\mathrm{CD}_{3} \mathrm{CN}, 298 \mathrm{~K}, 282.2 \mathrm{~Hz}, \delta[\mathrm{ppm}]\right):-79.2$ (s); IR (Nujol mull, $\mathrm{NaCl}$ plates, $\left.\mathrm{cm}^{-1}\right): 1963(\mathrm{w}), 1703(\mathrm{w}), 1629(\mathrm{~s}), 1542(\mathrm{~s})$,

Synthesis of $\left[\mathbf{P h P}(\mathbf{d m a p})_{2}\right][\mathrm{OTf}]_{2}$ : To a rapidly stirring solution of $\mathrm{PhPCl}_{2}(1.00 \mathrm{mmol}, 0.179 \mathrm{~g})$ and TMSOTf (2.00 mmol, $0.445 \mathrm{~g})$ in $5 \mathrm{~mL}$ DCM, dmap (4.00 mmol, $0.489 \mathrm{~g})$ was added, producing a pale pink solution. The solution was stirred for $1 \mathrm{hr}$ and then checked by ${ }^{31} \mathrm{P}\left\{{ }^{1} \mathrm{H}\right\}$ NMR spectroscopy. The solvent was removed under reduced pressure and washed with diethyl ether three times. The colourless powder was crystallized from $\mathrm{MeCN}$ layered with diethyl ether at $-26{ }^{\circ} \mathrm{C}$ for $48 \mathrm{hr}$, yielding pale peach crystals. Yield: $0.442 \mathrm{~g} \mathrm{(68} \mathrm{\% );} \mathrm{mp:}$ $159-161{ }^{\circ} \mathrm{C}$; EA [calc.]: C, 46.29; H, 4.33, N, 4.15 EA [found.]: C, 45.97; H, 4.42; N 4.05, ${ }^{1} \mathrm{H}$ NMR $\left(\mathrm{CD}_{2} \mathrm{Cl}_{2}, 298\right.$ $\mathrm{K}, 300 \mathrm{MHz}, \delta[\mathrm{ppm}]): 8.32-8.29\left(m, 4 \mathrm{H}, \mathrm{CH}_{\text {arom }}\right), 7.77-7.63\left(m, 5 \mathrm{H}, \mathrm{CH}_{\text {arom }}\right), 7.05-7.01\left(m, 4 \mathrm{H}, \mathrm{CH}_{\text {arom }}\right), 3.30$ 
(broad s, 12H, $\left.\mathrm{CH}_{3}\right) ;{ }^{13} \mathrm{C}\left\{{ }^{1} \mathrm{H}\right\} \mathrm{NMR}\left(\mathrm{CD}_{2} \mathrm{Cl}_{2}, 298 \mathrm{~K}, 75.4 \mathrm{MHz}, \delta[\mathrm{ppm}]\right)$ : $158.4(s), 143.6(s), 134.4(s), 131.1(s)$, $119.3(s), 110.1(s), 66.2(s), 41.3(s) ;{ }^{31} \mathrm{P}\left\{{ }^{1} \mathrm{H}\right\}$ NMR $\left(\mathrm{CD}_{2} \mathrm{Cl}_{2}, 298 \mathrm{~K}, 121.4 \mathrm{MHz}, \delta[\mathrm{ppm}]\right): 121.03(s) ;{ }^{19} \mathrm{~F}\left\{{ }^{1} \mathrm{H}\right\}$ $\operatorname{NMR}\left(\mathrm{CD}_{2} \mathrm{Cl}_{2}, 298 \mathrm{~K}, 282.2 \mathrm{~Hz}, \delta\right.$ [ppm]): -78.8 (s); IR (Nujol mull, NaCl plates, $\mathrm{cm}^{-1}$ ): 1645 (s), 1579 (s), 1533 (s), 1507 (s),

Synthesis of $\left[\mathbf{P h P}\left(\mathrm{SPCy}_{3}\right)_{2}\right][\mathbf{O T f}]_{2}$ : To a rapidly stirring solution of $\mathrm{PhPCl}_{2}(0.408 \mathrm{mmol}, 0.073 \mathrm{~g})$ and TMSOTf $(0.816 \mathrm{mmol}, 0.181 \mathrm{~g})$ in $5 \mathrm{~mL} \mathrm{DCM}, \mathrm{SPCy}_{3}(0.816 \mathrm{mmol}, 0.255 \mathrm{~g})$ was added, producing a colourless solution. The solution was stirred for $1 \mathrm{hr}$ and the solvent was removed under reduced pressure and washed with $\mathrm{Et}_{2} \mathrm{O}$ three times. The colourless powder product was crystallized from $\mathrm{DCM}$ layered with $\mathrm{Et}_{2} \mathrm{O}$ at room temperature. Yield: $0.240 \mathrm{~g}\left(57\right.$ \%); mp: $188-190^{\circ} \mathrm{C}$; EA [calc.]: C, 51.25; H, 6.94, N, 4.15 EA [found.]: C, 50.92; $\mathrm{H}, 7.16 ; \mathrm{N}<0.3,{ }^{1} \mathrm{H}$ NMR $\left(\mathrm{CD}_{3} \mathrm{CN}, 298 \mathrm{~K}, 300 \mathrm{MHz}, \delta[\mathrm{ppm}]\right): 8.13-7.62\left(m, 5 \mathrm{H}, \mathrm{CH}_{\text {arom }}\right), 2.76-1.20(m, 60 \mathrm{H}$, $\left.\mathrm{CH}_{2}\right) ;{ }^{13} \mathrm{C}\left\{{ }^{1} \mathrm{H}\right\} \mathrm{NMR}\left(\mathrm{CD}_{3} \mathrm{CN}, 298 \mathrm{~K}, 75.4 \mathrm{MHz}, \delta[\mathrm{ppm}]\right): 136.0(s), 134.5(s), 134.13(s), 131.8\left(d, J_{C P}=10.3 \mathrm{~Hz}\right)$, $36.0\left(d, J_{C P}=29.5 \mathrm{~Hz}\right), 28.6($ broad $s), 27.3\left(d, J_{C P}=13.1 \mathrm{~Hz}\right), 26.1(s) ;{ }^{31} \mathrm{P}\left\{{ }^{1} \mathrm{H}\right\} \mathrm{NMR}\left(\mathrm{CD}_{3} \mathrm{CN}, 298 \mathrm{~K}, 121.4\right.$ $\mathrm{MHz}, \delta[\mathrm{ppm}]): 73.7\left(d,{ }^{2} J_{P P}=21 \mathrm{~Hz}\right), 61.5\left(t,{ }^{2} J_{P P}=21 \mathrm{~Hz}\right) ;{ }^{19} \mathrm{~F}\left\{{ }^{1} \mathrm{H}\right\} \mathrm{NMR}\left(\mathrm{CD}_{3} \mathrm{CN}, 298 \mathrm{~K}, 282.2 \mathrm{~Hz}, \delta[\mathrm{ppm}]\right):-$ $79.2(s)$; IR (Nujol mull, $\mathrm{NaCl}$ plates, $\mathrm{cm}^{-1}$ ): 1580 (w),

Synthesis of $\left[\mathrm{PhP}\left(\mathrm{SePCy}_{3}\right)_{2}\right][\mathrm{OTf}]_{2}$ : To a rapidly stirring solution of $\mathrm{PhPCl}_{2}(0.380 \mathrm{mmol}, 0.068 \mathrm{~g})$ and TMSOTf (1.00 mmol, $0.222 \mathrm{~g})$ in $5 \mathrm{~mL} \mathrm{DCM}, \mathrm{SePCy}_{3}(0.950 \mathrm{mmol}, 0.341 \mathrm{~g})$ was added, producing a deep yellow solution. The solution was stirred for 30 minutes, becoming a pale yellow, and then checked by ${ }^{31} \mathrm{P}\left\{{ }^{1} \mathrm{H}\right\} \mathrm{NMR}$ spectroscopy. The solvent was removed under reduced pressure and washed with diethyl ether three times. The colourless powder product was crystallized from DCM layered with diethyl ether at $-26^{\circ} \mathrm{C}$ for 48 hours, producing pale yellow crystals. Yield: 0.402 g (94 \%); Melting Point: dec. $198{ }^{\circ} \mathrm{C}$; EA [calc.]: C, 46.98; H, 6.36, N, 0 EA [found.]: $\mathrm{C}, 47.16 ; \mathrm{H}, 6.52 ; \mathrm{N}<0.3,{ }^{1} \mathrm{H} \mathrm{NMR}\left(\mathrm{CD}_{2} \mathrm{Cl}_{2}, 298 \mathrm{~K}, 300 \mathrm{MHz}, \delta\right.$ [ppm]): $8.21-7.56\left(m, 5 \mathrm{H}, \mathrm{CH}_{\text {arom }}\right)$, $2.75-1.11\left(m, 60 \mathrm{H}, \mathrm{CH}_{2}\right) ;{ }^{13} \mathrm{C}\left\{{ }^{1} \mathrm{H}\right\} \mathrm{NMR}\left(\mathrm{CD}_{2} \mathrm{Cl}_{2}, 298 \mathrm{~K}, 75.4 \mathrm{MHz}, \delta[\mathrm{ppm}]\right): 134.4\left(d, J_{C P}=28.2 \mathrm{~Hz}\right), 130.8$ $\left(d,{ }^{2} J_{C P}=8.6 \mathrm{~Hz}\right), 123.4(s), 119.8(s), 35.7\left(d, J_{C P}=23.2 \mathrm{~Hz}\right), 28.8(s), 26.8\left(d, J_{C P}=12.3 \mathrm{~Hz}\right), 25.7(s) ;{ }^{31} \mathrm{P}\left\{{ }^{1} \mathrm{H}\right\}$ $\operatorname{NMR}\left(\mathrm{CD}_{2} \mathrm{Cl}_{2}, 298 \mathrm{~K}, 121.4 \mathrm{MHz}, \delta[\mathrm{ppm}]\right): 72.3\left(d,{ }^{2} J_{P P}=26 \mathrm{~Hz},{ }^{1} J_{P S e}=417 \mathrm{~Hz}\right), 48.9\left(t,{ }^{2} J_{P P}=26 \mathrm{~Hz}\right.$, broadening for the Se satellites); ${ }^{19} \mathrm{~F}\left\{{ }^{1} \mathrm{H}\right\}$ NMR $\left(\mathrm{CD}_{2} \mathrm{Cl}_{2}, 298 \mathrm{~K}, 282.2 \mathrm{~Hz}, \delta[\mathrm{ppm}]\right):-79.2(s) ;{ }^{77} \mathrm{Se}\left\{{ }^{1} \mathrm{H}\right\} \mathrm{NMR}$ $\left(\mathrm{CD}_{2} \mathrm{Cl}_{2}, 298 \mathrm{~K}, 68.2 \mathrm{~Hz}, \delta[\mathrm{ppm}]\right): 119\left(d d, J_{S e P}=416.5,133.5 \mathrm{~Hz}\right)$. 


\section{References}

[1] a) N. Burford, P. Losier, A. D. Phillips, P. J. Ragogna, T. S. Cameron, Inorg. Chem. 2003, 42, 1087-1091; b) N. Burford, H. A. Spinney, M. J. Ferguson, R. McDonald, Chem. Commun. 2004, 2696-2697; c) N. Burford, A. D. Phillips, H. A. Spinney, M. Lumsden, U. Werner-Zwanziger, M. J. Ferguson, R. McDonald, J. Am. Chem. Soc. 2005, 127, 3921-3927.

[2] a) P. A. Gray, Y.-y. Carpenter, N. Burford, R. McDonald, Dalton Trans. 2016, 45, 2124-2129; b) K. Schwedtmann, S. Schulz, F. Hennersdorf, T. Strassner, E. Dmitrieva, J. J. Weigand, Angew. Chem. Int. Ed. 2015, 54, 11054-11058; c) J. J. Weigand, K. O. Feldmann, F. D. Henne, J. Am. Chem. Soc. 2010, 132, 16321-16323; d) M. Azouri, J. Andrieu, M. Picquet, H. Cattey, Inorg. Chem. 2009, 48, 1236-1242.

[3] a) Á. Kozma, J. Rust, M. Alcarazo, Chem. - Eur. J. 2015, 21, 10829-10834; b) L. Gu, G. Gopakumar, P. Gualco, W. Thiel, M. Alcarazo, Chem. - Eur. J. 2014, 20, 8575-8578; c) K. Schwedtmann, M. H. Holthausen, K. O. Feldmann, J. J. Weigand, Angew. Chem. Int. Ed. 2013, 52, 14204-14208; d) R. Bashforth, A. J. Boyall, P. K. Coffer (née Monks), K. B. Dillon, A. E. Goeta, J. a. K. Howard, A. M. Kenwright, M. R. Probert, H. J. Shepherd, A. L. Thompson, Dalton Trans. 2012, 41, 1165-1172; e) K. B. Dillon, A. E. Goeta, J. a. K. Howard, P. K. Monks, H. J. Shepherd, A. L. Thompson, Dalton Trans. 2008, 1144; f) A. Schmidpeter, S. Lochschmidt, K. Karaghiosoff, W. S. Sheldrick, J. Chem. Soc., Chem. Commun. 1985, 1447.

[4] J. J. Weigand, S. D. Riegel, N. Burford, A. Decken, J. Am. Chem. Soc. 2007, 129, 7969-7976.

[5] J. Petuškova, M. Patil, S. Holle, C. W. Lehmann, W. Thiel, M. Alcarazo, J. Am. Chem. Soc. 2011, 133, 20758-20760.

[6] a) J. L. Dutton, P. J. Ragogna, Coord. Chem. Rev. 2011, 255, 1414-1425; b) C. A. Dyker, N. Burford, Chem. Asian J. 2008, 3, 28-36; c) J. J. Weigand, N. Burford, A. Decken, A. Schulz, Eur. J. Inorg. Chem. 2007, 3, 4868-4872; d) V. G. Nenajdenko, N. E. Shevchenko, E. S. Balenkova, I. V. Alabugin, Chem. Rev. 2003, 103, 229-282; e) T. A. Engesser, M. R. Lichtenthaler, M.

Schleep, I. Krossing, Chem. Soc. Rev. 2016, 45, 789-899; f) A. P. M. Robertson, P. A. Gray, N. Burford, Angew. Chem. Int. Ed. 2014, 53, 6050-6069; g) F. D. Henne, A. T. Dickschat, F. Hennersdorf, K. O. Feldmann, J. J. Weigand, Inorg. Chem. 2015, 54, 6849-6861.

[7] S. S. Chitnis, A. P. M. Robertson, N. Burford, B. O. Patrick, R. McDonald, M. J. Ferguson, Chem. Sci. 2015, 6, 6545-6555.

[8] a) K. L. Bamford, A. P. M. Robertson, H. A. Jenkins, B. O. Patrick, N. Burford, Can. J. Chem. 2015, 93, 375-379; b) K. Tani, K. Matsuyama, S. Kato, K. Yamada, H. Mifune, Bull. Chem. Soc. Jpn. 2000, 73, 1243-1252.

[9] M. H. Stewart, M. J. A. Johnson, J. W. Kampf, Organometallics 2007, 26, 5102-5110.

[10] J. E. McDonough, A. Mendiratta, J. J. Curley, G. C. Fortman, S. Fantasia, C. C. Cummins, E. V. Rybak-Akimova, S. P. Nolan, C. D. Hoff, Inorg. Chem. 2008, 47, 2133-2141.

[11] P. Pyykkö, M. Atsumi, Chem. - Eur. J. 2009, 15, 186-197.

[12] S. Alvarez, Dalton Trans. 2013, 42, 8617.

[13] K. A. Kerr, P. M. Boorman, B. S. Misener, J. G. H. v. Roode, Can. J. Chem. 1977, 55, 30813085 .

[14] P. L. Folkins, B. R. Vincent, D. N. Harpp, Tetrahedron Lett. 1991, 32, 7009-7012.

[15] J. A. Davies, S. Dutremez, A. A. Pinkerton, Inorg. Chem. 1991, 30, 2380-2387.

[16] N. A. Barnes, S. M. Godfrey, R. T. A. Halton, I. Mushtaq, R. G. Pritchard, S. Sarwar, Dalton Trans. 2006, 1517-1523.

[17] G. M. Sheldrick, Acta Cryst. Sect. A 2008, 64, 112-122. 
Graphical Abstract

Compounds with the generic formula $\left[\mathrm{PhPL}_{2}\right][\mathrm{OTf}]_{2}$ contain cations that represent complexes of the phenylphosphine dication expanding the scope of coordination complexes involving phosphorus as an acceptor.

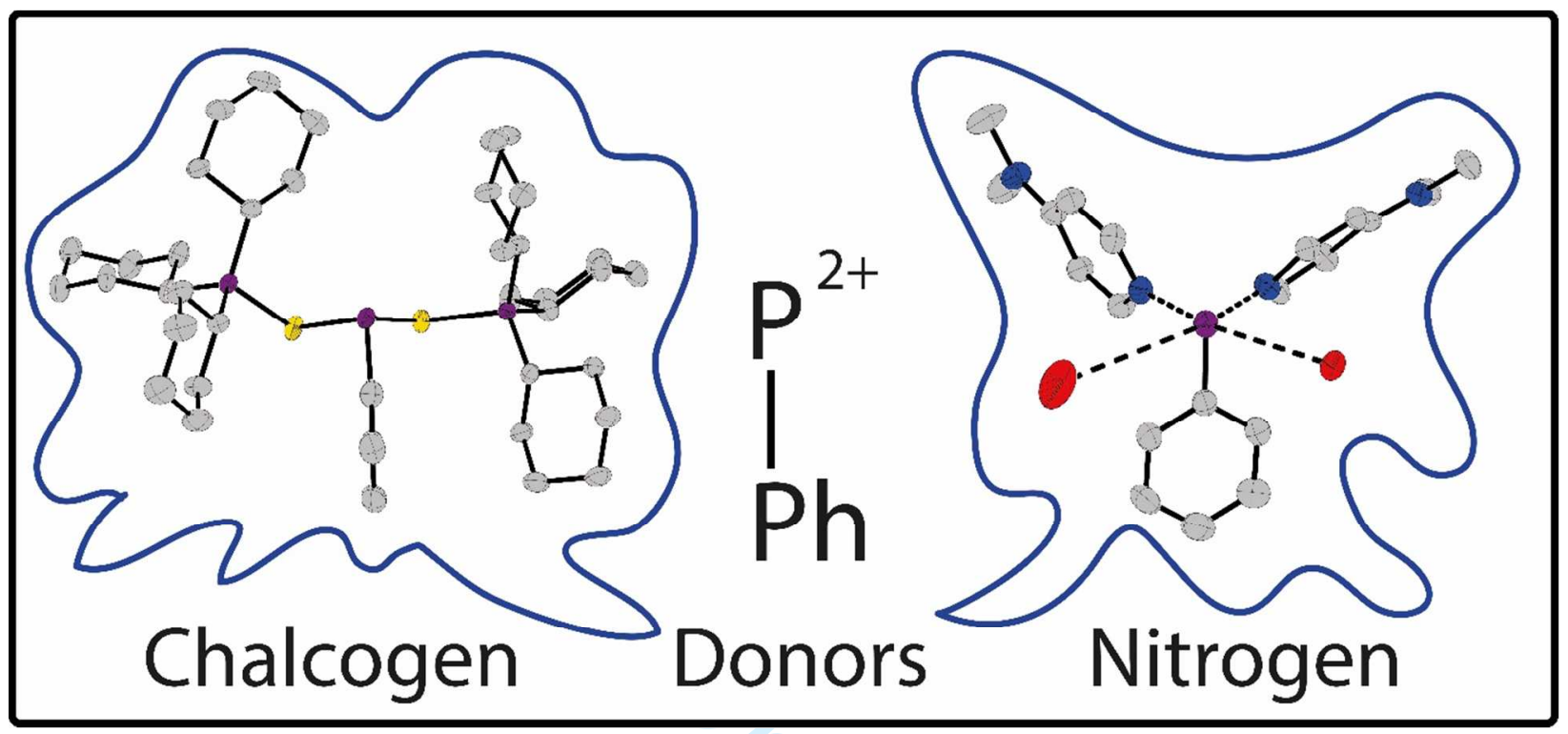

